\title{
Niveles de sedentarismo de una institución educativa en Popayán, Colombia
}

\author{
Sedentarism levels of an educational institution in Popayan, Colombia
}

Luz Marina Chalapud-Narváez ${ }^{*}$ orcid.org/0000-0003-4047-7105

Nancy Janeth Molano-Tobar ${ }^{2}$ orcid.org/0000-0003-1953-4101

Magdi Yanette Ordoñez-Fernández² orcid.org/0000-0003-3786-6687

1 Facultad de Educación, Programa Entrenamiento Deportivo. Corporación Universitaria Autónoma del Cauca. Popayán, Colombia

2 Facultad de Ciencias Naturales, Exactas y de la Educación. Universidad del Cauca. Popayán, Colombia

\section{Resumen}

Introducción: La niñez y la adolescencia son etapas de desarrollo del ser humano susceptibles a cambios en los estilos de vida donde se pueden intensificar comportamientos sedentarios durante las actividades de la vida diaria. Objetivo: Determinar los niveles de sedentarismo de los estudiantes de una Institución Educativa de Popayán-Colombia. Materiales y métodos: Estudio cuantitativo de tipo no experimental, con diseño descriptivo de corte transversal. La muestra fue estratificada simple obtenida bajo fórmula probabilística compuesta por 92 estudiantes, $51,1 \%$ del sexo masculino, con edad promedio de 12,5 años \pm 3,21 años. Se evaluó el nivel de sedentarismo mediante el test del cajón de Pérez-Rojas-García. Resultados: El 79,3\% de la población practica deporte o actividad física, respecto al índice de masa corporal el 72,83\% están en normopeso y de acuerdo al test de sedentarismo el $41,3 \%$ son sedentarios severos y el $43,5 \%$ son sedentarios moderados. Conclusión: La población escolar presenta un factor de riesgo para la salud asociado a comportamientos sedentarios.

Palabras clave: Salud; estilo de vida; actividad física; estudiantes. (Fuente: DeCS, Bireme).

\begin{abstract}
Introduction: Childhood and adolescence are stages of development of the human being susceptible to changes in lifestyles where sedentary behaviors can be intensified during activities of daily living. Objective: To determine the sedentarism levels of the students of an Educational Institution of Popayán-Colombia. Materials and methods: A quantitative study of nonexperimental type, with descriptive cross-sectional design. The simple stratified sample was obtained under the probabilistic formula composed of 92 students, $51.1 \%$ of which were male, with an average age of $12.5 \pm 3.21$ years. The level of sedentary lifestyle was assessed by the Pérez-Rojas-García drawer test. Results: 79.3\% of the population practice sports or physical activity, the body mass index was $72.83 \%$ that represents normal weight and according to the sedentary lifestyle test $41.3 \%$ are severe sedentary and $43.5 \%$ are moderate sedentary. Conclusion: The school population presents a risk factor for health associated with sedentary behavior.
\end{abstract}

Key words: Health; life style; physical activity; students. (Source: DeCS, Bireme).

\footnotetext{
*Autor de correspondencia

Luz Marina Chalapud Narváez

e-mail: luz.chalapud.n@uniautonoma.edu.co
} 


\section{Introducción}

La niñez y la adolescencia son etapas de desarrollo del ser humano susceptibles a cambios en los estilos de vida, influenciados por la moda, entorno social y familiar(1), que repercuten sobre la "calidad de vida relacionada con la salud infanto-juvenil"(2), que se determina, principalmente, por la alimentación y la baja actividad física (AF). Es así que en el mundo, al menos un $60 \%$ de la población no realiza actividad física $(\mathrm{AF})^{(3)}$, además la inactividad se intensifica con los comportamientos sedentarios durante las actividades de la vida diaria (AVD).

Carson, et al.(4), definen la conducta sedentaria como la realización de actividades de bajo gasto energético en posición sedente o reclinado, ante ello la Organización Mundial de la Salud (OMS) plantea que "el sedentarismo afecta a más del $60 \%$ de la población mundial" $(5,6)$, y que esto se asocia a las dinámicas sociales actuales, uso de aparatos tecnológicos, la inseguridad que disminuye la realización de actividades al aire libre y las AVD que se realizan sentados, estos comportamientos condicionan los factores de aparición del sedentarismo, con consecuencias directas sobre la salud(7).

Uno de los grupos poblacionales más afectados son los niños y adolescentes, ya que son más propensos a adquirir costumbres sedentarias ${ }^{(8)}$, viéndose reflejado en las jornadas escolares donde deben permanecer sentados la mayor parte del tiempo. Cárdenas, et al.(9), mencionan que "niños y jóvenes invierten en su educación en promedio 360 horas al año, 8 horas/día", lo cual fomenta la conducta sedentaria que se acrecienta por el uso de medios de transporte motorizados, la ausencia de infraestructura adecuada para la práctica de $\mathrm{AF}^{(10)}$, la inseguridad. Sumado a ello, los compromisos laborales de los padres, que de acuerdo a la Organización Internacional del Trabajo (OIT), el $47,3 \%$ de las mujeres en el mundo trabajan, condicionando el ingreso temprano de los niños al sistema educacional, pasando gran parte del día en los jardines infantiles y escuelas"(5), fomentando una generación de conductas escolares ya mencionadas.

Con lo anterior, se adiciona el panorama alimentario donde el niño es inducido a ingerir desde la escuela y por influencias publicitarias de la televisión "chucherías y golosinas"(11) de dudoso valor nutricional, generalmente con alto contenido calórico que no contribuyen a una dieta saludable, recurriendo al consumo de alimentos con exceso de grasas y carbohidratos, haciendo del sedentarismo, un círculo vicioso, que retroalimenta los factores de riesgo de la población infantil(12).

Determinar los niveles de sedentarismo en la población infantil permitirá generar o fortalecer programas de $\mathrm{AF}$ en el ámbito escolar, para influir positivamente en la salud de los futuros adultos, situación que hasta el momento está generando un alto costo en la salud pública de un país como Colombia, como lo reportan Guerrero, et al.(13), quienes indican que el sedentarismo es un predictor de enfermedades crónicas no trasmisibles (ECNT) como la obesidad, hipertensión arterial y diabetes entre otras; situación comentada por el estudio de Hernández(14), que demostró la asociación entre la inactividad física y la presencia de ECNT, hecho que añade una preocupación a la situación actual de la población infantil, que tiene una tendencia hacia el sedentarismo y puede tener manifestaciones de prehipertensión o hipertensión arterial.

Ferreira, et al.(15), expresan claramente que la hipertensión infantil es una tendencia presente y fuera de tener asociado los factores de riesgo ya conocidos en el adulto (predisposición genética, desarrollos intrauterinos, estrato socioeconómico, tabaquismo y obesidad abdominal), se evidencian los factores extrínsecos como la $\mathrm{AF}$ y la conducta sedentaria, que están conllevando a incremento en los niveles de riesgo cardiovasculares en niños. Por tal razón, se planteó como objetivo determinar el nivel de sedentarismo de los estudiantes de una institución educativa de básica primaria y secundaria de la ciudad de Popayán.

\section{Materiales y métodos}

El estudio cuantitativo de tipo no experimental, con un diseño descriptivo y de corte transversal.

\section{Participantes}

La población estuvo constituida por 189 estudiantes. La muestra fue probabilística estratificada simple y correspondió a 124 estudiantes, la muestra se definió con un nivel de confianza del $95 \%$ y un margen de error del 5\%. Finalizaron el estudio 92 estudiantes, $47(51,1 \%)$ hombres y $45(48,9 \%)$ mujeres con una edad promedio de $12,5 \pm 3,21$ años en un rango de edad de 7 a 18 años, quienes cumplieron con los 
siguientes criterios de inclusión: estar legalmente matriculados en la institución educativa, firmar el consentimiento informado, presentar el asentimiento firmado por el representante legal. Del estudio fueron excluidos los estudiantes con discapacidad física, que no se presentaron a la evaluación y que se retiraron voluntariamente del mismo.

\section{Instrumento}

Se aplicó el test de sedentarismo de Pérez-Rojas García, validado por otros estudios(13,16,17). El test consiste en subir y bajar un escalón de $25 \mathrm{~cm}$ de altura, durante 3 minutos, aplicando tres cargas con ritmos progresivos $(17,26$ y 34 pasos por minuto). Se aplica cada carga durante 3 minutos y se descansa 1 minuto entre ellas. Para ejecutar el test se debe tomar la presión arterial y el pulso en reposo, definir el $65 \%$ de la Frecuencia Cardiaca Máxima (FCM) del evaluado en un minuto. El resultado se divide entre 4, para llevarlo a la frecuencia en 15 seg., esa cifra se convierte en el límite de asimilación de las tres cargas físicas posibles a aplicar $(17,26$ y 34 pasos por minuto). Teniendo en cuenta los resultados y la aplicación de la fórmula, se clasifica al individuo en sedentario severo si no vence la primera carga, sedentario moderado si vence la primera carga, pero no la segunda, activo si vence la segunda carga, pero no la tercera o muy activo si vence la tercera carga(13).

Los parámetros antropométricos tenidos en cuenta para la medición fueron el índice de masa corporal (IMC) que, se calcula dividiendo el peso de una persona en kilos por el cuadrado de su talla en metros $\left(\mathrm{kg} / \mathrm{m}^{2}\right)^{(18)}$, y para la clasificación se tuvo en cuenta las tablas de IMC para la edad de niños(as) y adolescentes de 5 a 18 años de edad de la OMS, cuya clasificación es: desnutrición severa ( $<-3$ DS), desnutrición moderada ( $\geq-3$ DS a $<-2$ DS), normal ( $\geq$ $-2 \mathrm{DS} \mathrm{a} \leq+1 \mathrm{DS})$, sobrepeso ( $>+1 \mathrm{DS} \mathrm{a} \leq+2 \mathrm{DS}) \mathrm{y}$ obesidad $(>+2$ DS)(18,19).

\section{Procedimiento}

La recolección de la información se realizó en el primer semestre del año 2016, utilizando una encuesta que permitió identificar aspectos sociodemográficos, como también aspectos antropométricos e indicadores de salud. Se solicitó a los niños la autorización por medio del asentimiento informado firmado por sus padres o tutor. El test se aplicó durante las clases de Educación Física y los participantes tuvieron la posibilidad de desistir de su participación en el estudio.

\section{Análisis de datos}

Se utilizó el programa SPSS versión 24.0 (SPSS Inc., Chicago, EE.UU.). Se realizó análisis univariado aplicando medidas de tendencia central y de dispersión de la misma manera se aplicaron mediciones para evidenciar la uniformidad del grupo con la prueba Kolmogorov-Smirnov. Para el análisis bivariado se tuvo en cuenta un nivel de significancia estadística valor de $\mathrm{p} \leq 0,05$ y se aplicó Chi cuadrado de Pearson.

\section{Consideraciones éticas}

El estudio fue aprobado por el Comité de Ética del Sistema de Investigación de la Corporación Universitaria Autónoma del Cauca, quien revisó que los procedimientos de la investigación siguieran las consideraciones éticas de la declaración de Helsinki y la Resolución 8430 de 1993, clasificando el estudio en riesgo mínimo. Además, los participantes firmaron el asentimiento y consentimiento informado según el caso.

\section{Resultados}

La mayoría de los participantes fueron de quinto y séptimo grado y el $60,9 \%$ de los estudiantes pertenecían al estrato socioeconómico medio. Con relación a los estilos de vida el 79,3\% de los estudiantes manifestaron practicar AF, y la mayoría, $40(54,8 \%)$, que respondieron que si realizan $\mathrm{AF}$ son de grados iniciales, con una práctica semanal de 2 días (19,6\%), 3 días (28,3\%), una duración de 30 a 60 minutos (37\%) y más de 60 minutos $(31,5 \%)$ por sesión; siendo los hombres quienes más practican actividad física o deporte, ya que se encontró 40 $(43,47 \%)$ hombres que manifestaron realizar AF vs $33(35,86 \%)$ mujeres. En relación al uso de aparatos tecnológicos en el tiempo libre se evidenció que el $77,2 \%$ usa celular y el $62 \%$ ve televisión, y se evidenció que los escolares dedican más de 2 horas diarias al uso de pantallas (Tabla 1).

De acuerdo a los parámetros antropométricos, la media del peso fue de $43,2 \pm 12,4 \mathrm{Kg}$, de la talla $1,49 \pm 0,14 \mathrm{~m}$ y del IMC $18,9 \pm 12,9$ (Tabla 2). La clasificación del IMC tuvo una tendencia al peso normal $(72,83 \%)$, seguida del sobrepeso $(23,9 \%)$, y la desnutrición severa, desnutrición moderada y obesidad presentaron una distribución del 1,09\% cada una. Al comparar está variable con el sexo se observa la misma distribución de peso normal tanto para niños como niñas y con relación al grado de 
escolaridad no hay diferencia entre los estudiantes de grados superiores como inferiores.

Tabla 1. Caracterización sociodemográfica y de estilos de vida de los estudiantes de una institución educativa de Popayán

\begin{tabular}{|c|c|c|}
\hline Variable & $\mathrm{N}=92$ & $\%$ \\
\hline \multicolumn{3}{|l|}{ Grado escolar } \\
\hline Tercero & 17 & 18,5 \\
\hline Quinto & 21 & 22,8 \\
\hline Séptimo & 20 & 21,7 \\
\hline Noveno & 16 & 17,4 \\
\hline Décimo & 18 & 19,6 \\
\hline \multicolumn{3}{|l|}{ Estrato socioeconómico } \\
\hline Bajo & 2 & 2,2 \\
\hline Medio bajo & 33 & 35,9 \\
\hline Medio & 56 & 60,9 \\
\hline Medio alto & 1 & 1,1 \\
\hline \multicolumn{3}{|c|}{ Práctica de actividad física o deporte } \\
\hline $\mathrm{Si}$ & 73 & 79,3 \\
\hline No & 19 & 20,7 \\
\hline \multicolumn{3}{|c|}{$N^{\circ}$ días de práctica a la semana } \\
\hline 1 & 15 & 16,3 \\
\hline 2 & 18 & 19,6 \\
\hline 3 & 26 & 28,3 \\
\hline 4 & 6 & 6,5 \\
\hline 5 & 8 & 8,7 \\
\hline No aplica & 19 & 20,7 \\
\hline \multicolumn{3}{|l|}{ Horas de práctica al día } \\
\hline$<30$ minutos & 9 & 9,8 \\
\hline Entre 30 y 60 minutos & 34 & 37,0 \\
\hline$>60$ minutos & 29 & 31,5 \\
\hline \multicolumn{3}{|l|}{ tiempo libre tv } \\
\hline $\mathrm{Si}$ & 57 & 62,0 \\
\hline No & 35 & 38,0 \\
\hline \multicolumn{3}{|l|}{ Tiempo libre pc } \\
\hline $\mathrm{Si}$ & 48 & 52,2 \\
\hline & 44 & 47,8 \\
\hline \multicolumn{3}{|l|}{ Tiempo libre celular } \\
\hline $\mathrm{Si}$ & 71 & 77,2 \\
\hline No & 21 & 22,8 \\
\hline
\end{tabular}

$T V=$ televisión, $P C=$ computadora

Tabla 2. Caracterización antropométrica de los estudiantes

\begin{tabular}{|c|c|c|c|c|c|c|}
\hline Variable & $\frac{\pi}{\overbrace{0}^{\circ}}$ & 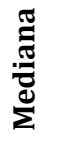 & $\frac{\pi}{\tilde{D}}$ & $\tilde{a}$ & $\stackrel{\varrho}{\stackrel{\Xi}{\Xi}}$ & 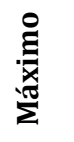 \\
\hline Peso (kg.) & 43,2 & 44,3 & 48,9 & 12,4 & 19,0 & 74,4 \\
\hline Talla (m) & 1,49 & 1,50 & 1,35 & 146 & 1,17 & 1,84 \\
\hline IMC & 18,9 & 18,6 & 23,3 & 12,9 & 12,2 & 27,4 \\
\hline
\end{tabular}

IMC: índice de masa corporal; DS: desviación estándar

Referente a los niveles de sedentarismo se encontró que el $84,77 \%$ de los estudiantes son sedentarios, la población de escolares activa y muy activa sólo está representada en el 15,23\% (Tabla 3).

Tabla 3. Clasificación del nivel de sedentarismo de los estudiantes

\begin{tabular}{lrrr}
\hline \multicolumn{1}{c}{ Nivel sedentarismo } & $\mathbf{N}=\mathbf{9 2}$ & \multicolumn{1}{c}{ \% } \\
\hline Sedentarismo severo & & 38 & 41,30 \\
Sedentarismo moderado & 40 & 43,47 \\
Activo & 13 & 14,13 \\
Muy activo & 1 & 1,1 \\
\hline
\end{tabular}

Los resultados del cruce de la variable nivel de sedentarismo con las variables sociodemográficas, de hábitos saludables y antropométricas no evidenciaron asociación estadísticamente significativa $(\mathrm{p} \leq 0,05)$ (Tabla 4$)$, pero se encontraron diferencias numéricas que mostraron que los hombres tienden a ser más sedentarios, 42 (45,65\%) hombres frente a $36(39,1 \%)$ mujeres. También, se encontró que hay mayor población sedentaria en secundaria 46 (50\%), comparándolo con la población de primaria $32(34,78 \%)$.

Tabla 4. Resultados del cruce de la variable sedentarismo con las variables sociodemográficas, de estilos de vida y antropométricas de los estudiantes

\begin{tabular}{lcr}
\hline \multicolumn{1}{c}{ Variables cruzadas } & $\begin{array}{c}\text { Valor } \\
\mathbf{X}^{2}\end{array}$ & $\begin{array}{c}\text { Sig. } \\
\text { Asintótica }\end{array}$ \\
\hline $\begin{array}{l}\text { Nivel de sedentarismo*sexo } \\
\text { Nivel de sedentarismo*grado }\end{array}$ & 6,513 & 0,089 \\
$\begin{array}{l}\text { escolar } \\
\text { Nivel de sedentarismo*estrato }\end{array}$ & 6,312 & 0,900 \\
$\begin{array}{l}\text { socioeconómico } \\
\text { Nivel de sedentarismo*clasificación }\end{array}$ & 8,571 & 0,702 \\
$\begin{array}{l}\text { IMC } \\
\text { Nivel de sedentarismo*práctica de }\end{array}$ & 9,519 & 0,739 \\
$\begin{array}{l}\text { AF o deporte } \\
\text { Nivel de sedentarismo*días de } \\
\text { práctica de AF o deporte }\end{array}$ & 10,482 & 0,391 \\
$\begin{array}{l}\text { Nivel de sedentarismo*horas de } \\
\text { práctica al día }\end{array}$ & 4,243 & 0,574 \\
$\begin{array}{l}\text { Nivel de sedentarismo*tiempo libre } \\
\text { en TV }\end{array}$ & 2,180 & 0,643 \\
$\begin{array}{l}\text { Nivel de sedentarismo* tiempo } \\
\text { libre en PC }\end{array}$ & 2,930 & 0,403 \\
$\begin{array}{l}\text { Nivel de sedentarismo* tiempo } \\
\text { libre en celular }\end{array}$ & 8,320 & 0,842 \\
\hline$X^{2}=$ Chi cuadrado de Pearson, IMC = índice de masa corporal & \\
\hline
\end{tabular}

\section{Discusión}

Los resultados mostraron que el sexo no determina las conductas sedentarias, hecho que difiere de investigaciones recientes, que evidenciaron mayor grado de sedentarismo en las mujeres ${ }^{(20,21)}$. Con 
relación al grado escolar se encontró que los estudiantes de secundaria son más sedentarios, lo anterior se asemeja a lo encontrado en varias investigaciones como las de Abarca et al., y la de Buhring, et al.,(8,22) resaltando un porcentaje mayor de sedentarios en los adolescentes de secundaria, afirmando que con el pasar del tiempo el sedentarismo en los jóvenes se ha incrementado.

A diferencia de investigaciones con poblaciones similares se encontró que los escolares del estudio, están en el rango del IMC normal, lo que resulta ser determinante en los estilos de vida saludable de la población, ya que un aumento del IMC se relaciona con la poca práctica de $\mathrm{AF}$ y el incremento de sobrepeso y obesidad (23). Por otra parte, en el estudio realizado por Siquier, et al.(24), encontraron resultados similares con relación al IMC de la población.

Los escolares en su mayoría pertenecen al estrato socioeconómico medio, sin presentar relación con el sedentarismo, lo anterior se asemeja a la investigación realizada por Alcibíades, et al.(25), quienes plantearon que es importante enfatizar en la asociación entre sedentarismo y estrato socioeconómico, ya que esta relación está sustentada en los estilos de vida más inactivos y los cambios en los hábitos en algunos estratos socioeconómicos.

Aunque la mayoría de los escolares dicen practicar $\mathrm{AF}$, que puede identificarse como un hábito saludable, presentan comportamientos sedentarios marcados(26), entre ellos la falta de realización de actividad física adecuada y el tiempo dedicado al uso de pantallas. Hay una tendencia de mayor actividad en los niños en numerosas investigaciones se ha demostrado que los escolares presentan un bajo nivel de $\mathrm{AF}$, y que son los niños quienes practican mayor $\mathrm{AF}(5,26,27)$ con relación a las niñas, lo que puede explicarse desde el concepto que las madres conducen a que las niñas tengan conductas de quietud o de recato, propiciando que los niños salgan a jugar con sus pares y no con las niñas(28-30).

Complementando esta variable de práctica de AF se demostró que quienes más la realizan son los escolares en grados iniciales, lo que es explicado por Trejo, et al.(31), al denotar que la práctica de $\mathrm{AF}$ disminuye con el edad y se debe en gran parte al desarrollo de caracteres sexuales secundarios que conllevan a que el juego y la $\mathrm{AF}$ queden en un segundo plano.
Respecto a la frecuencia semanal de $\mathrm{AF}$ y la dedicación en horas, se encontró que los escolares cumplen con las recomendaciones mundiales de la OMS(3,31-34), que son cumplir con un mínimo de 60 minutos diarios de AF, mínimo 3 veces por semana, similar a los procesos investigativos adelantados por Ramos, et al.(35), quien infiere la posibilidad de que los escolares no presenten problemas de salud relacionadas con ECNT, sin embargo, difieren de la investigación de Simons, et al.(36), que identificó que los niños no realizan AF superior a 30 minutos a la semana, la cual coincide con la clase de educación física. Al respecto, la Encuesta Nacional de la Situación Nutricional - ENSIN, encontró que sólo el 31,1\% de la población de 5 a 12 años y el 13,4\% de la población entre 13 y 17 años, cumplen con las recomendaciones de $\mathrm{AF}^{(37) \text {. }}$

Se ha planteado en esta investigación que la práctica de la AF tiene como limitante las horas que los escolares dedican a ver televisión, usar computadoras y celular, disminuyendo el tiempo a realizar prácticas saludables, lo anterior se asemeja a los hallazgos de Prieto, et al.(29), quienes indican que a mayor tiempo de exposición a los aparatos tecnológicos es menor el tiempo de AF. Adicionalmente, Martínez, et al.(38), refieren que el papel de la publicidad televisiva incita a que los individuos consuman alimentos con alto contenido energético y bebidas azucaradas, indudablemente poco saludables para la salud. Según, la $\operatorname{ENSIN}^{(37)}$ el $67,6 \%$ de la población entre 5 y 12 años y el 76,6\% de la población entre 13 y 17 años, dedican tiempo excesivo frente a pantallas. Por su parte, Del Águila(39), describió que la prevalencia del sobrepeso y la obesidad en niños y adolescentes constituyen un problema de salud pública emergente en el Perú, e instó sobre la necesidad de prevenir oportunamente el desarrollo de enfermedades crónicas, garantizando calidad de vida durante la adultez.

La mayoría de la población objeto de estudio es sedentaria, hallazgo importante, ya que la edad de afectación de esta problemática es crucial en la instauración o no, de prácticas saludables, además con base a estos resultados se puede inferir la posibilidad de desarrollar tempranamente factores de riesgo para la aparición de $\operatorname{ECNT}(37,38)$. Por otra parte, en el contexto local no se ha realizado investigaciones en poblaciones infantiles o adolescentes, pero sí en universitarios, encontrando que el $97 \%$ de la población es sedentaria(13), situación similar a la reportada por Vidarte, et al.,(16) en su 
estudio con población entre 18 y 60 años, donde más del 70\% de la población es sedentaria.

No se presenta relación estadísticamente significativa con el nivel de sedentarismo, lo cual difiere de otras investigaciones ${ }^{(13,40)}$ que manifiestan que al cruzar los datos de niveles de AF y conducta sedentaria existe una relación inversamente proporcional, además plantean que las personas que no realizan $\mathrm{AF}$ representan la mayor parte de la población sedentaria.

\section{Conclusiones}

La mayoría de los escolares son sedentarios, evidenciando la presencia de un factor de riesgo para la salud temprana, que puede fomentar la aparición de enfermedades crónicas no transmisibles como la obesidad, la diabetes, entre otras.

No se encontró relación de los niveles de sedentarismo con las variables sociodemográficas, antropométricas y de estilos de vida.

Se resalta que la mayoría de los escolares son de estrato socioeconómico medio, que no permite evidenciar la diferencia que pudiese existir en los diferentes niveles económicos, de acuerdo a las posibilidades de adquisición de hábitos o estilos de vida que propicien el comportamiento sedentario.

\section{Agradecimientos}

Se agradece a la Corporación Universitaria Autónoma del Cauca por propiciar el desarrollo de procesos de investigación. A la Universidad del Cauca, cuya alianza fortaleció el proceso de esta investigación. Un especial agradecimiento a los estudiantes Felipe Lozano y Mauricio Torres, pilares fundamentales en este proceso. Código de identificación del proyecto: 0239.

\section{Conflicto de intereses}

Los autores no reportan conflictos de intereses de ninguna clase.

\section{Referencias}

1. Núñez HP, Campos N, Holst-Schumacher I, Alfaro-Mora FV. Las creencias de la docente de educación física sobre la obesidad en la niñez de edad escolar. Rev Electrónica Educ. 2013;17:5-30.

2. Orgiles M, Sanz I, Piqueras JA, Espada JP. Differences in eating habits and physical activity in a sample of preadolescent depending on their weight category. Nutr Hosp. 2014;30(2):306-13.

3. Aguilar-Cordero MJ, Ortegón-Piñero A, Mur-Villar $\mathrm{N}$, Sánchez-García JC, Verazaluce JG, García IG, et al. Programas de actividad física para reducir sobrepeso y obesidad en niños y adolescentes; revisión sistemática. Nutr Hosp. 2014;30(4):727-40.

4. Carson V, Wong SL, Winkler E, Healy GN, Colley RC, Tremblay MS. Patterns of sedentary time and cardiometabolic risk among Canadian adults. Prev Med (Baltim). 2014;65:23-7.

5. Cappelacci MC, Alfaro TO, Artigas FL, Muñoz CS. Relación entre estado nutricional, nivel de actividad física y desarrollo psicomotor en preescolares. Nutr Hosp. 2014;30(6):1313-8.

6. Chalapud-Narváez LM, Escobar-almario A. Actividad física para mejorar fuerza y equilibrio en el adulto mayor. Univ. Salud. 2017;19(1):94-101.

7. Abreu de Carvalho C, de Almeida-Fonsêca PC, Pacheco de Oliveira F, de Almeida Coelho A, Pinheiro-Machado S. Fatores sociodemográficos associados a prática de exercício físico, uso do computador, assistir à TV e jogar videogame entre adolescents. Adolesc e Saude. 2015;12(2):17-28.

8. Abarca - Sos A, Zaragoza Casterad J, Generelo Lanaspa E, Julián Clemente J. Comportamientos sedentarios y patrones de actividad física en adolescentes. Rev Int Med y Ciencias la Act Física y el Deport. 2010;10(39):410-27.

9. Cárdenas SD, Martínez FG, Vergara KA. Niveles de actividad física asociados a factores sociodemograficos, antropométricos y conductuales en universitarios de Cartagena (Colombia). Salud Uninorte. 2014;30(3):405-17.

10. Arango Forero G, Bringué Sala X, Sádaba Chalezquer C. La generación interactiva en colombia: adolescentes frente a la Internet, el celular y los videojuegos. Anagramas. 2010;9(17):45-6.

11. Tirado F, Barbancho FJ, Prieto J, Moreno A. Influencia de los hábitos televisivos infantiles sobre la alimentación y el sobrepeso (II). Rev Cubana Enferm. 2014;20(3):1-4.

12. Lavielle-Sotomayor $P$, Pineda-Aquino $V$, Jáuregui-Jiménez $O$, Castillo-Trejo M. Actividad física y sedentarismo: Determinantes sociodemográficos, familiares y su impacto en la salud del adolescente. Rev salud pública. 2014;16(2):161-72.

13. Guerrero Pepinosa NY, Muñoz Ortiz RF, Muñoz Martínez AP, Pabón Muñoz JV, Ruiz Sotelo DM, Sánchez DS. Nivel de sedentarismo en los estudiantes de fisioterapia de la fundación universitaria María Cano, Popayán. Hacia la promoción la salud. 2015;20(2):77-89.

14. Hernando Sanz MA. Calidad de vida, Educación Física y Salud. Rev Española Pedagog. 2006;64(235):453-63.

15. Ferreira A, Barbosa H, Siani A, Barba G, Veidebaum T, Tornaritis $\mathrm{M}$, et al. Incidence of high blood pressure in children - Effects of physical activity and sedentary behaviors : The IDEFICS study High blood pressure, lifestyle and children. Int J Cardiol. 2015;180:165-70.

16. Vidarte Claros JA, Àlvarez CV, Aduen Angel JI. Niveles de sedentarismo en población entre 18 y 60 años: Sincelejo (Colombia). Salud Uninorte. 2015;31(1):70-7.

17. Vidarte-Claros J a., Vélez-Álvarez C, Parra-Sánchez JH. Niveles de sedentarismo en población de 18 a 60 años. Manizales, Colombia. Rev salud pública [Internet]. 2012;14(3):417-28. Available from: http://www.scielosp.org/pdf/rsap/v14n3/v14n3a05.pdf

18. Organización Mundial de la Salud. Obesidad y sobrepeso [Internet]. Ginebra: OMS; 2017. Available from: http://www.who.int/mediacentre/factsheets/fs311/es/ 
19. Food and Nutrition Technical Assistance FANTA III. Tablas de IMC y tablas de IMC para la edad, de niños(as) y adolescentes de 5 a 18 años de edad y tablas de IMC para adultos(as) no embarazadas, no lactantes $\geq 19$ años de edad. [Internet]. Washington; 2013. Available from: https://www.fantaproject.org/sites/default/files/resources /FANTA-BMI-charts-Enero2013-ESPANOL_0.pdf

20. Silva-Strempler JA, Cuevas-Vargas PG, Espinosa-Márquez CE, García-Torres G. Sedentarismo y obesidad en estudiantes universitarios de primer semestre estudio comparativo. Rev Cuid. 2012;1(1):64-70.

21. Verela MT, Duarte C, Salazar IC, Lema LF, Tamayo JA. Actividad física y sedentarismo en jóvenes universitarios de Colombia: prácticas, motivos y recursos para realizarlas. Colomb Med. 2011;42(3):269-77.

22. Buhring BK, Oliva MP, Bravo CC. Determinación no experimental de la conducta sedentaria en escolares. Rev Chil Nutr. 2009;36(1):23-30.

23. Díaz S, González F, Arrieta K. Niveles de actividad física asociados a factores sociodemograficos, antropométricos y conductuales en universitarios de Cartagena (Colombia). Salud Uninorte. 2014;30:405-17.

24. Siquier-Coll J, Collado-Martín Y, Sánchez-Puente M, GrijotaPérez F, Pérez Quintero M, Bartolomé-Sánchez I, et al. Estudio comparativo de las variables determinantes de la condición física y salud entre jóvenes deportistas y sedentarios del género masculino. Nutr Hosp. 2018;35(3):689-697.

25. Alcibíades-Bustamante V, Seabra AF, Garganta RM, Maia JA. Efectos de la actividad física y del nivel socioeconómico en el sobrepeso y obesidad de escolares, Lima este 2005. Rev Peru Med Exp Salud pública. 2007;24(2):121-8.

26. Ruiz G, De Vicente E, Vegara J. Comportamiento sedentario y niveles de actividad en una muestra de estudiantes y trabajadores universitarios. J Sport Heal Res. 2012;4(1):8392.

27. Lopez-Munera R, Santos MAC, Navarro A, Arévalo JM, García $\mathrm{F}$, Latorre P. Determinantes sociodemográficos y nivel de actividad física en la población de la provincia de Jaén mayor de 18 años. Retos Nuevas Tendencias en Educ Fis Deport y Recreacion. 2016;20(41):13-6.

28. Torres-luque G, Carpio E, Sánchez AL, Luisa M, Sánchez Z. Niveles de condición física de escolares de educación primaria en relación a su nivel de actividad física y al género. Retos Nuevas Tendencias en Educ Fis Deport y Recreacion. 2014;20(41):17-22.
29. Prieto-Benavides DH, Correa-Bautista JE, Ramírez-Vélez R. Niveles de actividad física, condición física y tiempo en pantallas en escolares de Bogotá, Colombia: Estudio FUPRECOL. Nutr Hosp. 2015;32(5):2184-2192.

30. Beltrán VJ, Sierra AC, Jiménez A, González-Cutre D, Martínez C, Cervelló E. Diferencias según género en el tiempo empleado por adolescentes en actividad sedentaria y actividad fisica en diferentes segmentos horarios del día. Retos Nuevas Tendencias en Educ Fis Deport y Recreación. 2017;(31):3-7.

31. Trejo PM, Jasso S, Mollinedo FE, Lugo LG. Relación entre actividad física y obesidad en escolares Relation. Rev Cuba Med Gen Integr. 2012;28(1):34-41.

32. Organización Mundial de la Salud. Guía de actividad física [Internet]. Ginebra: OMS; 2016. Available from: http://whqlibdoc.who.int/publications/2010/9789243599 977_spa.pdf

33. Jakicic J, Marcus B, Gallagher K, Napolitano M, Lang W. Effect of exercise duration and intensity on weight loss in overweight, sedentary women: a randomized trial. JAMA. 2003;290:1323-30.

34. Pate R, Pratt M, Blair S. Physical activity and public health: a recommendation from the Centers for Disease Control and Prevention and the American College of Sport Medicine. JAMA. 1995;273:402-7.

35. Ramos C, González JA, López JD. Actividad física y adiposidad en la población de Neiva. Rev Educ Física y Deport. 2013;32: 481-9.

36. Simons-Morton GB, Taylor CW, Zinder AS, Huang WI. The physical activity of fifth-grade students during physical education classes. Am J Public Health. 1993;8(2):262-4.

37. Ministerio de Salud y Protección Social. Bogotá, Colombia: Encuesta Nacional de la Situación Nutricional - ENSIN 2015; 2015.

38. Martínez-Moyá M, Navarrete-Muñoz EM, García M, GiménezMonzo D, González-Palacios S, Valera-Gran D, et al. Asociación entre horas de televisión, actividad física, horas de sueño y exceso de peso en población adulta joven. Gac Sanit. 2014;28(3):203-8.

39. Del Águila-Villar CM. Obesidad en el niño: factores de riesgo y estrategias para su prevención en Perú. Rev Peru Med Exp Salud Pública. 2017;34(1):113-8.

40. Felden ÉPG, Filipin D, Barbosa DG, Andrade RD, Meyer C, Beltrame TS, et al. Adolescentes com sonolência diurna excessiva passam mais tempo em comportamento sedentário. Rev Bras Med do Esporte. 2016;22(3):186-90. 